\title{
'Keeping the kids at home' can limit the persistence of contagious pathogens in social animals
}

\author{
Lucile Marescot $^{1,2}$ (I) | Mathias Franz ${ }^{1}$ (D) | Sarah Benhaiem ${ }^{1}$ (I) | Heribert Hofer ${ }^{1,3,4}$ | \\ Cédric Scherer ${ }^{1}$ (D) | Marion L. East ${ }^{1}$ | Stephanie Kramer-Schadt ${ }^{1,5}$ (D)
}

${ }^{1}$ Leibniz Institute for Zoo and Wildlife Research, Berlin, Germany

${ }^{2}$ CEFE, CNRS, University Montpellier, University Paul Valéry, EPHE, Montpellier, France

${ }^{3}$ Department of Veterinary Medicine, Freie Universität Berlin, Berlin, Germany

${ }^{4}$ Department of Biology, Chemistry, Pharmacy, Freie Universität Berlin, Berlin, Germany

${ }^{5}$ Institute of Ecology, Technische Universität Berlin, Berlin, Germany

Correspondence

Sarah Benhaiem

Email: benhaiem@izw-berlin.de

\section{Present address}

Mathias Franz, Institute for Biology, Freie Universität Berlin, Königin-Luise-Strasse 1-3, Berlin, 14195, Germany

\section{Funding information}

Leibniz-Institut für Zoo- und

Wildtierforschung; Leibniz-Gemeinschaft, Grant/Award Number: SAW-2015-IZW-1 440 AquaVir and SAW-2018-IZW-3EpiRank; Agence Nationale de la Recherche, Grant/Award Number: ANR-16-CE02-0007; Deutsche Forschungsgemeinschaft, Grant/ Award Number: EA 5/3-1 and KR 4266/2-1

Handling Editor: Andy Fenton

\section{Abstract}

1. Social networks are considered to be 'highly modular' when individuals within one module are more connected to each other than they are to individuals in other modules. It is currently unclear how highly modular social networks influence the persistence of contagious pathogens that generate lifelong immunity in their hosts when between-group interactions are age dependent. This trait occurs in social species with communal nurseries, where juveniles are reared together for a substantial period in burrows or similar forms of containment and are thus in isolation from contact with individuals in other social groups.

2. Our main objective was to determine whether, and to what extent, such agedependent patterns of between-group interactions consistently increased the fade-out probability of contagious pathogens that generate lifelong immunity in their hosts. We hypothesised that in populations of species where juveniles are raised in communal nurseries, a high proportion of recovered adults in a group would form a 'protective barrier' around susceptible juveniles against pathogen transmission, thereby increasing the probability of epidemic fade-out in the population.

3. To test this idea, we implemented a spatially implicit individual-based susceptibleinfected-recovered (SIR) model for a large range of generic host and pathogen traits.

4. Our results indicated that (a) the probability of epidemic fade-out was consistently higher in populations with communal nurseries, especially for highly contagious pathogens (high basic reproduction number, $R_{0}$ ) and (b) communal nurseries can counteract the cost of group living in terms of infection risk to a greater extent than variation in other traits.

5. We discuss our findings in relation to herd immunity and outline the importance of considering the network structure of a given host population before implementing management measures such as vaccinations, since interventions focused on

Lucile Marescot, Mathias Franz and Sarah Benhaiem have a shared first authorship. Marion East and Stephanie Kramer-Schadt have a shared last authorship.

This is an open access article under the terms of the Creative Commons Attribution License, which permits use, distribution and reproduction in any medium, provided the original work is properly cited.

(c) 2021 The Authors. Journal of Animal Ecology published by John Wiley \& Sons Ltd on behalf of British Ecological Society 
individuals with high between-group contact should be particularly effective for controlling pathogen spread in hosts with communal nurseries.

\section{KEYWORDS}

altricial, between-group contact, communal burrows, community structure, crèche, herd immunity, outbreak, SIR model

\section{1 | INTRODUCTION}

Pathogens transmitted by direct contact or physical proximity, for example via aerosols, may imperil populations of species that live at high densities, including humans, livestock and some wildlife species (Altizer et al., 2003). Understanding the patterns of transmission and the mechanisms of persistence of such pathogens in group living species is thus of key relevance for human health and wildlife conservation. The structure of social contact networks in group living species may critically influence the spread and persistence of contagious pathogens (Kappeler et al., 2015). For example, if a social network contains a few central individuals, who are exceptionally well-connected to others, then these individuals can act as superspreaders and their presence can strongly increase the likelihood and extent of an epidemic as well as the persistence of pathogens (Lloyd-Smith et al., 2005).

A social network can be characterised by its modularity, that is, its degree of division into modules such as social groups (e.g. clans, prides or troops) or subgroups such as age classes in eusocial insects (Stroeymeyt et al., 2018) or colonies in seabirds (Nunn et al., 2006 Wanelik et al., 2017). Generally, a social network is considered to be 'highly modular' when individuals within one module are more connected to each other than they are to individuals in other modules (Newman, 2006). Highly modular social networks may generally reduce infection risk in group living animals (Griffin \& Nunn, 2012; Nunn et al., 2015). This view has been recently challenged by a study which found that the beneficial effects of modularity on epidemic fade-out are restricted to cases of exceptionally high modularity, rarely observed in nature (Sah et al., 2017).

The objective of this study was to use a conceptual model to determine whether and how an age-dependent pattern of betweengroup interactions, which increases social network modularity, affected pathogen spread and persistence. An age-dependent pattern of between-group interactions is interesting as it is expected to occur in social species with communal nurseries, that is where typically altricial juveniles shelter together in communal burrows, dens, lairs or crèches within group territories or home ranges for extended periods of time, or emerge from such structures shortly after birth but stay in their vicinity for a considerable period of their life span, thereby not encountering members of other groups. Such species include spotted hyenas Crocuta crocuta (Kruuk, 1972), yellow-bellied marmots Marmota flaviventris (Wey \& Blumstein, 2010) or grey wolves Canis lupus (Mech \& Boitani, 2003, see Table S1 for further examples). The persistence of pathogens which induce lifelong immunity following infection may be reduced in networks in which young have no contact with members of other groups. Such pathogens, which include many viruses in the genus Morbillivirus such as measles and canine distemper virus, mumps in the genus Rubulavirus or rubella in the genus Rubivirus (Morris et al., 2015), rely on the birth of new susceptible hosts and their transmission to juveniles to persist in a host population (Lloyd-Smith et al., 2005).

Communal nurseries could affect pathogen persistence via at least two, non-exclusive, mechanisms. First, as a rather intuitive effect, by limiting contacts of juveniles with members of other groups, communal nurseries should reduce the overall contact rate in the population, thereby decreasing the basic reproduction number $\left(R_{0}\right)$ of a pathogen, the expected number of secondary infections that a single infected host is responsible for in a population of susceptible individuals (Anderson \& May, 1991) and thus the probability of pathogen spread. Second, they could increase the chance of epidemic fade-out by depriving the pathogen of access to susceptible individuals during the late stage of an epidemic. When most adults in the population have become immune and thus herd immunity occurs (see e.g. Anderson \& May, 1985), the chance of pathogen spread to mostly susceptible young animals would be substantially curtailed. In other words, communal nurseries may increase the efficiency of a protective barrier in groups composed of recovered adults, which emerges naturally when social hosts are infected with pathogens that confer lifelong immunity, thereby increasing the probability of epidemic fade-out.

We developed a generic individual-based model to investigate this second mechanism and test the prediction that the probability of epidemic fade-out increases in populations (networks) of social species with communal nurseries. We used a susceptible-infectedrecovered (SIR) framework to describe pathogen transmission and host population dynamics, and first developed a baseline scenario in which the rates of between-group contact were identical for individuals of all age classes, assuming homogeneous mixing. We then investigated a scenario in which individuals below a given age threshold could not contact members of other groups, so their rates of between-group contact were set to zero. We considered these two extremes for ease of interpretation and to identify the principal mechanism. Because communal nurseries decrease $R_{0}$, we adjusted the infection rate in the baseline scenario in such a way that the value of $R_{0}$ was always identical in both scenarios, thereby ensuring that the emergence of a more efficient immunity barrier was directly related to communal nurseries and not to its mediated effect on $R_{0}$. We investigated both scenarios for every specific combination of key host and pathogen traits known to influence pathogen transmission dynamics. 


\section{2 | MATERIALS AND METHODS}

\section{1 | Model description}

The model description followed the ODD (Overview, Design concepts and Details) protocol for individual-based and agent-based models (Grimm et al., 2006, 2010). The model was implemented in NetLogo (v.6.1.1) and is available on GitHub (https://github.com/ EcoDynIZW/Marescot_Franz_Benhaiem_2021_JAnimEcol). We used R v. 4.0.2. (R Core Team, 2020), Rstudio v1.3.959 (RStudio Team, 2020) and the packages DPLYR (Wickham, François, et al., 2019), GGPLOT2 (Wickham, 2016), GGTEXT (Wilke, 2020), hERE (Müller, 2017), PATCHWORK (Pedersen, 2019), RPART (Therneau \& Atkinson, 2018), SHOWTEXt (Qiu, 2020) and TIDYVERSE (Wickham, Averick, et al., 2019).

\subsection{1 | Purpose}

The purpose of our conceptual model was to assess whether and how an age-dependent pattern of between-group interactions, which increases social network modularity, consistently increased the probability of epidemic fade-out across a large range of host and pathogen life history traits. This trait occurs in social species with communal nurseries where juveniles have no contact with members of other social groups during their development. We modelled a contagious pathogen spreading through physical contact and/or spatial proximity, for example, via aerosols, and inducing lifelong immunity following infection. All model parameters below can easily be changed to tailor the model to host-specific life histories and pathogen traits.

\subsection{2 | Entities, state variables and scales}

Individuals and modules were regarded as entities. Each individual was characterised by three state variables updated on a weekly basis: age, epidemiological status (susceptible S, infected I or recovered R, i.e. immune) and the time spent infected. Modules represented social groups, which consisted of a certain number of individuals which could change over time because of mortality, dispersal and immigration. Week was the unit of time considered here but any other unit of time (e.g. days, years) could in principle be applied.

\subsection{3 | Processing and scheduling}

The model included two main processes, namely pathogen transmission dynamics and host population dynamics. At each time step, the following sequence of processes occurred: (a) pathogen transmission, (b) death, (c) reproduction, (d) dispersal/immigration and (e) ageing of host individuals. We modelled the following two classic functional responses (parameter transmission in Table 1): density-dependent transmission, in which the per capita contact rate between susceptible and infected individuals depends on host population density so that the transmission rate increases with density, and frequency-dependent transmission, where it does not, so that transmission rate does not vary with host density (Begon et al., 2002). Both types are possible when pathogens spread through social contact and/or proximity (Begon et al., 2002; Borremans et al., 2017). Abbreviated model parameters (in italics below) are defined in Table 1, where their simulation values are also provided.

During pathogen transmission, the force of infection was influenced by (a) the age at first between-group contact, age_contact, (b) the number (for density-dependent transmission) or proportion (for frequency-dependent transmission) of infected individuals and (c) the contact ratio $\theta$, the ratio of between-group to within-group contact, which considers different levels of between-group mixing. Overall network modularity therefore depended on both age_contact and $\theta$. Contact ratio $\theta$ defined how much two individuals from different groups interacted in comparison with two individuals of the same group. That is, if $\theta=1$, all individuals interacted equally, independent of their social group affiliation (lower network modularity, homogeneous mixing). Conversely, if $\theta=0$, social interactions occurred exclusively within groups (higher network modularity). In the baseline scenario, we assumed equal contact between all age classes, with contact rates within and between groups defined by $\theta$. In the communal nursery scenario, when young individuals started with between-group contacts only from a given age onwards (here age_contact $=52$ weeks), we assumed contact rates within and between groups defined by $\theta$. In such a scenario, for a given $\theta$, the overall modularity in the network was thus always higher than in the baseline scenario.

Note that we did not model social interactions explicitly, that is individual movements and encounters (either via direct contact or physical proximity) were modelled implicitly through $\theta$. Such a formalisation allows us to capture a wide range of potential pathogens and modes of transmission while simultaneously keeping the number of parameters in the model to a minimum. Pathogen traits such as virulence $\alpha$, pathogen infectivity infectivity, $R_{0}$, and maximum infection length infect_length also affected transmission.

The second process (death) included two forms of mortality: the host intrinsic mortality $(1-\phi$, with $\phi$ being the survival probability) and the host mortality induced by the pathogen (virulence $\alpha$ ). Individuals who survived over the maximum infection length (infect length) gained immunity for the rest of their lives.

The third process (reproduction) was regulated by the carrying capacity $K$ of the host. Births occurred only in groups that were below the local breeding capacity defined by the maximum group size. The probability that an individual older than age_repro (here age repro $=104$ weeks) gave birth to one offspring was given by repro. For simplicity, hereafter we use 'juveniles' for individuals younger than age_repro and 'adults' for individuals older than age_repro.

The fourth process (dispersal/ immigration) described individuals which left their natal group and immigrated into other groups as they became reproductively mature.

In the last process (ageing), we updated the individual's state variable age as well as the time span-infected counter. 
TAB LE 1 Host and pathogen-specific parameters in the individual-based model. The numbers in square brackets represent the values explored for each parameter that was varied in order to assess the sensitivity of epidemic fade-out probabilities to changes in parameters. The unit of time was week and state variables were updated on a weekly basis. We chose a given set of host and pathogen trait values that ensured the emergence and maintenance of an epidemic (and prevented the immediate extinction of the pathogen) in order to test our hypothesis

\begin{tabular}{|c|c|c|}
\hline Parameter & Description & Simulation values [values] \\
\hline$K$ & Carrying capacity of the population & 1,000 \\
\hline age_contact & $\begin{array}{l}\text { Age at first between-group contact (baseline: } 0 \text {, i.e. direct contact, communal } \\
\text { nursery: 52) }\end{array}$ & {$[0,52]$} \\
\hline$\phi$ & $\begin{array}{l}\text { Per step (week) survival probability (resulting in yearly survival probabilities equal to } \\
{[0.60,0.75,0.90] \text { ) }}\end{array}$ & {$[0.991,0.995,0.998]$} \\
\hline repro & Probability of reproducing (resulting in a fertility of one to three offspring p.a.) & {$[0.01,0.025,0.05]$} \\
\hline$\theta$ & $\begin{array}{l}\text { Contact ratio, that is the ratio between the rate of between-group contact and the } \\
\text { rate of within-group contact }\end{array}$ & {$[*, 0.1,0.5,1]$} \\
\hline infectivity & $\begin{array}{l}\text { Infectivity rate, that is the ability of the pathogen to infect the host (adjusted to } \\
\text { obtain similar } R_{0} \text { values for baseline and communal nursery scenarios). Simulation } \\
\text { values for } R_{0} \text { are provided here }\end{array}$ & {$[0.5,1,2,5,10,20]$} \\
\hline$\alpha$ & Virulence or case fatality, that is the probability of the host to die from an infection & {$[0,0.05,0.1]$} \\
\hline infect_length & Infection length & {$[10,20,30]$} \\
\hline transmission & Type of transmission (frequency vs. density dependent) & - \\
\hline
\end{tabular}

${ }^{*}$ We did not model the case of $\theta=0$, as this would imply that the pathogen is contained within one group and always dies out.

\subsection{4 | Initialisation}

The initialisation of the age of individuals was determined by a random exponential distribution with a mean set to the age at first reproduction (age_repro, Table 1). All groups were started at a certain group size and all individuals were set as susceptible, except one who was set as infected. Pathogen spread started stochastically with this infected individual chosen randomly in the initial population. The total population was at a carrying capacity of 1,000 individuals at the beginning of the simulations to make simulation runs comparable. The initial number of groups depended on the initialised number of individuals per group.

\subsection{5 | Input data}

This theoretical model did not use any empirical input.

\subsection{6 | Submodels}

\section{Pathogen transmission}

For individuals who reached or exceeded their age at first between group contact, transmission could occur both within (termed 'inside') and between (termed 'other') groups. Both sources of infection were considered in the calculation of the force of infection $\beta_{i, \text { age } \geq \text { age_contact }}$ of an individual $i$ for frequency-dependent transmission,

$$
\beta_{i, \text { age } \geq \text { age_contact }}=\left[\frac{I_{\text {inside }, t}}{N_{\text {inside }, t}}+\theta \times \frac{I_{\text {other }, t}}{N_{\text {other }, t}}\right] \times \text { infectivity },
$$

and for density-dependent transmission,

$$
\beta_{i, \text { age } \geq \text { age_contact }}=\left[I_{\text {inside }, t}+\theta \times I_{\text {other }, t}\right] \times \text { infectivity } .
$$

In both equations the first term in brackets represents the contact rate between a susceptible individual from a group with infected individuals from the same group at a time step $t\left(l_{\text {inside, }}\right)$. The second term in brackets represents the contact rate between a susceptible individual from a group with infected individuals from other groups $\left(l_{\text {other, },}\right)$. This second term depended on the contact ratio $\theta$, hence accounted for contact rates between individuals from different groups. Both terms were then multiplied by the infectivity of the pathogen infectivity, describing the probability that a susceptible individual became infected after exposure to the pathogen. In (1), $N_{\text {inside, }}$ and $N_{\text {other, }}$ represent the abundances within a given group and in other groups respectively.

For a susceptible individual $i$, the probability of becoming infected $p_{i}$ was given by the cumulative distribution function of the exponential distribution per time step: 


$$
P_{i, \text { age } \geq \text { age_contact }}=1-1 / \exp \left(\beta_{i, \text { age } \geq \text { age_contact }}\right) .
$$

For a susceptible individual $i$ of age <age_contact, transmission could occur within groups only. The probability of becoming infected depended on the infectivity of the pathogen and the risk of exposure to an infected individual. For frequency-dependent transmission, the force of infection $\beta_{i, \text { age }}$ age_contact was:

$$
\beta_{i, \text { age }<\text { age_contact }}=\left[\frac{I_{\text {inside, }}}{N_{\text {inside, }, t}}\right] \times \text { infectivity, }
$$

for density-dependent transmission,

$$
\beta_{i, \text { age }<\text { age_contact }}=\left[I_{\text {inside, } t}\right] \times \text { infectivity. }
$$

Each susceptible juvenile $i$ therefore had a probability of becoming infected $p_{i}$ as:

$$
p_{i, \text { age }<\text { age_contact }}=1-\frac{1}{\exp \left(\beta_{i, \text { age }<\text { age }- \text { contact }}\right)} .
$$

When an individual was infected, its epidemiological status changed from susceptible to infected and the counter for the time spent infected was set to one (for the first unit of time of infection), and then increased in each subsequent unit of time of infection. If the individual had survived the entire duration of the infection period (infect_length), its epidemiological status changed from infected to recovered. Pathogen clearance occurred when individuals were free of infection and still alive at the end of the infection period infect_length, that is when they entered the recovered state.

\section{Death}

At each time step, all individuals died with a probability of $1-\phi$, with $\phi$ being the survival probability, which captured causes of intrinsic mortality. In addition, infected individuals died with a probability of $\alpha$, the virulence of the pathogen.

\section{Reproduction}

We assumed density-dependent reproduction. Reproduction only occurred if the group size was below the carrying capacity of groups, that is the group_size threshold (Table 1). If reproduction occurred, then at every unit of time each adult older than age_repro gave birth to a single offspring with a probability equal to repro. All individuals were born as susceptible, independently of the epidemiological status of their parent.

\section{Dispersal}

We modelled primary dispersal, so all individuals left their natal group when they reached their reproductive maturity age_repro and moved to a randomly selected group (which included extinct groups).

\section{Ageing}

The process of ageing updated the individual's state variable age as well as the time span-infected counter, which was the time since an individual became infected.

\section{2 | Model analyses}

We assessed whether communal nurseries consistently increased the probability of epidemic fade-out across a range of host and pathogen life-history traits (2,916 combinations of parameters in total), while keeping $R_{0}$ identical between the baseline and the communal nursery scenario. To do so, we designed a multifactorial simulation experiment. We varied the simulation values of the parameters specific to host life history: survival $\phi$, probability of reproducing for reproductive individuals repro, group size group_size and contact ratio $\phi$ (Table 1 ). In order to verify that a potential beneficial effect of communal nurseries on epidemic extinction probability occurred irrespective of the breeding regime, we additionally ran a scenario with pulsed birth events simulating seasonal reproduction (breeding_regime, Table 1). We first determined the total number of individuals born per temporal cycle (here, 1 year), and then distributed this number during a quarter of the temporal cycle (here, during a 3-month period).

We also considered variation in the simulation values for parameters characterising the pathogen: virulence $\alpha$, infection length infect_length and $R_{0}$ which we calculated analytically (see Supporting Information). We adjusted infectivity infectivity between communal nursery and baseline scenarios so that both types of networks had similar $R_{0}$, all else being equal. We ran each simulation over 10 years and recorded whether the epidemic persisted or faded out. We repeated each parameter combination 30 times to examine the probability of epidemic fade-out. We plotted the average epidemic fade-out probability for communal nursery and baseline scenarios across all parameter combinations and for each value of $R_{0}$.

To determine whether variation in the age at first between-group contact, which differed between both scenarios, had the highest impact on the probability of epidemic fade-out, we compared epidemic fade-out probabilities calculated for the extreme values of each parameter, holding all other parameters equal. To increase robustness in the comparison of fade-out probabilities between both scenarios, we filtered the results by running a Fisher's exact test for each paired scenario. We tested whether differences in epidemic fade-out probabilities were significant and plotted the distributions of differences between epidemic fade-out probabilities only for paired scenarios with a $p$-value $<0.05$.

Finally, we ran a regression tree with the $R$ package RPART (Therneau \& Atkinson, 2018), using the ANOVA method, to partition the difference in epidemic fade-out probabilities between communal nursery and baseline scenarios. We used this approach to detect under which specific conditions (trait values) communal nurseries showed the greatest effect-provided this trait showed any effect at all (Breiman et al., 1984). The method consisted of splitting the parameter space into binary groups, which differed in terms of difference in epidemic fade-out probabilities with a minimum deviance of the standard deviation of the epidemic fade-out in those groups. 


\section{3 | RESULTS}

\section{1 | Effects of communal nurseries on epidemic fade-out probability}

As theory predicts, epidemics faded out at $R_{0}<1$, and did so regardless of the type of between-group interactions. The probability of epidemic fade-out was consistently higher in hosts with communal nurseries than in the baseline scenario, and this difference increased with increasing $R_{0}$ (Figure 1). This was true also for hosts with seasonal reproduction, even though the effect of communal nurseries was globally less important, particularly at high values of $R_{0}$ (Figure $\mathrm{S} 1$ ).

Age at first between-group contact was the parameter with the highest influence on epidemic fade-out probability. The difference in epidemic fade-out probabilities between the communal nursery and baseline scenarios was indeed greater than the difference in epidemic fade-out probabilities between the two most extreme values of any other parameter (Figure 2b, panel 'Age at first between-group contact').

The positive effects of communal nurseries for the host population in terms of epidemic fade-out probability were generally more important at high values of $R_{0}$ and for host species with a high fertility (see branches on the right side of Figure 3 ). Given this set of conditions, these positive effects were particularly elevated (a) for pathogens with a short period of infection and density-dependent transmission (0.70, 0.50 and 0.38, Figure 3), (b) in social networks composed of small groups affected by pathogens with a long period of infection and low virulence $(0.45$, Figure 3) and (c) networks with high contact ratio, frequencydependent transmission and a short period of infection $(0.36$, Figure 3).

\section{2 | SIR dynamics}

There were important differences between communal nursery and baseline scenarios (Figure 4) in how the number of individuals in the susceptible, infected and recovered epidemiological states changed over the first 10 years (520 weeks).

When looking at a single epidemic event in the communal nursery scenario, a new pool of susceptible juveniles emerged in the population a few weeks after initialisation to form a peak, followed by an increase in the number of susceptible adults (Figure 4a). The epidemic faded out from the host population approximately 50 weeks after the initialisation, in terms of the number of infected juveniles and adults (Figure 4b). This corresponds approximately to the age at which juveniles in the first cohort began to contact (potentially infected) members of other groups (age_contact $=52$ weeks, Table 1). The host population rapidly reached a stage in which all adults became recovered (approximately 27 weeks in Figure 4c). The last infected individuals were juveniles, and those surviving the infection became recovered adults (Figure 4c). Consequently, a few weeks after pathogen extinction and population turnover, the new pool of susceptible juveniles emerged in the population (Figure 4a). The number of recovered adults declined over time as they were not fuelled anymore by infected juveniles who would have turned into recovered adults (Figure 4c).

When looking at the same epidemic event in the baseline scenario, the host population was never composed of a large number of susceptible individuals (Figure $4 d$ ) and the pathogen persisted for at least 10 years after the peak of infected juveniles and adults (Figure 4e). Despite a high number of recovered adults, the pathogen was maintained in the host population at a prevalence of approximately $2 \%$ (Figure $4 \mathrm{e}$ ). Although a large portion of adults was recovered and did not contribute to transmission (Figure 4f), the pathogen

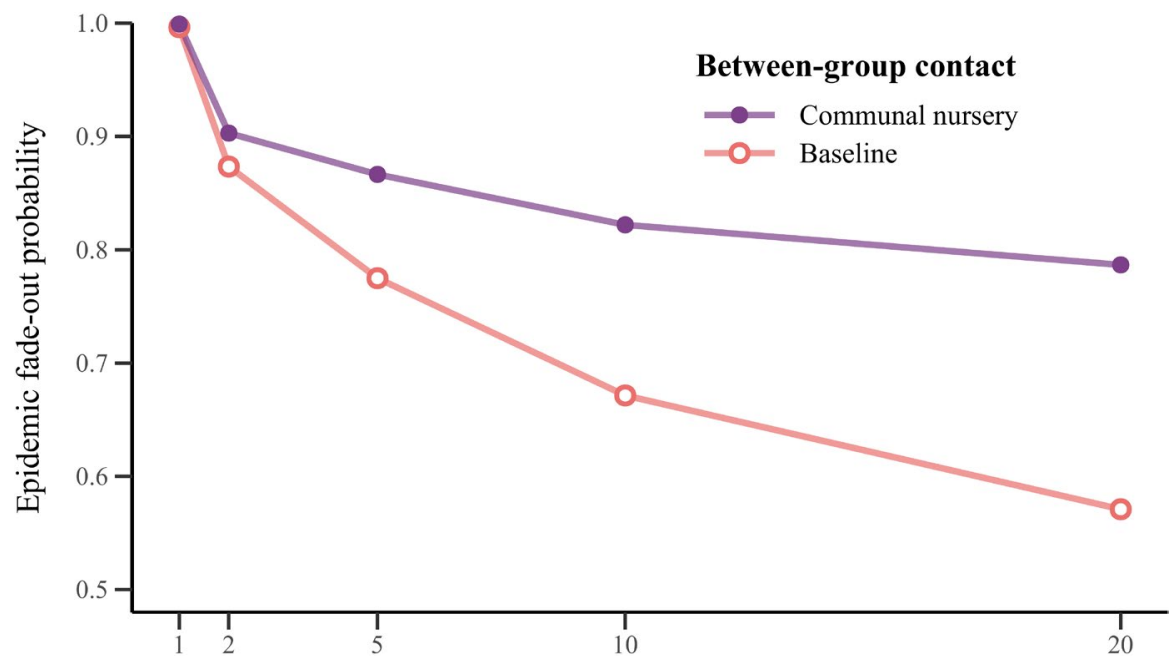

Basic reproduction number $R_{0}$
FIGURE 1 Probability of epidemic fade-out as a function of the basic reproduction number $R_{0}$ in social host populations with communal nurseries, where juveniles have no contact with members of other groups during their development (communal nursery scenario, purple), and age-independent between-group contacts (baseline, pink), for all possible combinations of host and pathogen traits 


\section{(a) Pathogen-related traits}
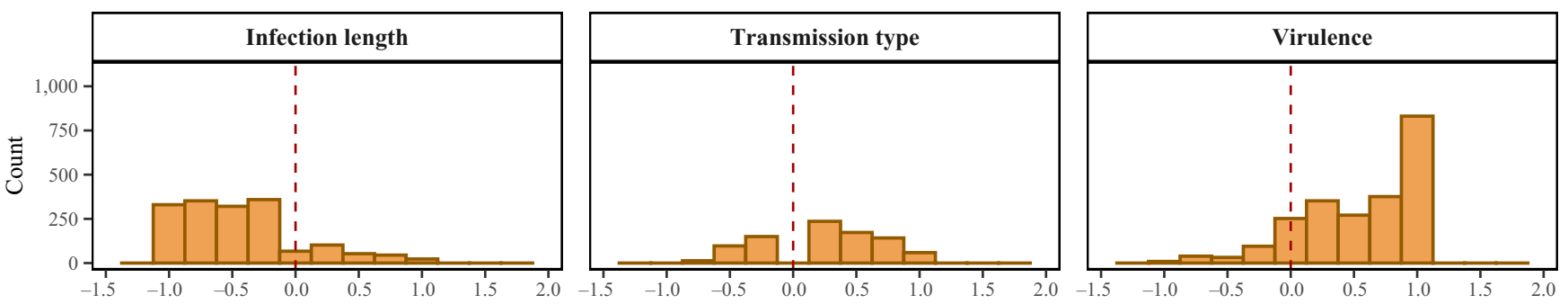

(b) Host-related traits
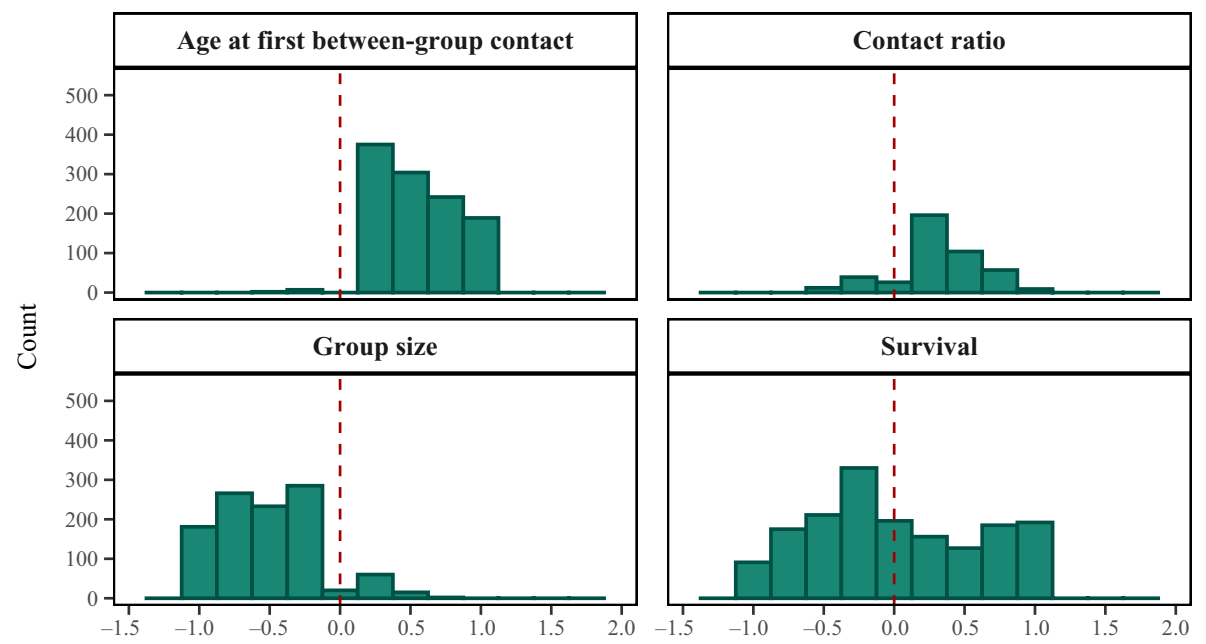

Difference in fade-out probabilities

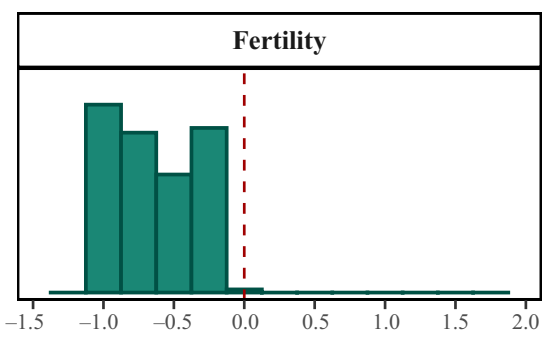

FIGURE 2 Number of simulations in relation to the difference in epidemic fade-out probabilities between two extreme values of each parameter for (a) pathogen and (b) host-related traits, all else being equal. The extreme values were (a) infection length (infect_length = 30 , infect_length $=10$ ), transmission type (frequency vs. density) and virulence $(\alpha=0.05, \alpha=0.1)$; (b) age at first between-group contact (52 weeks for the communal nursery scenario, 0 for the baseline scenario), contact ratio $(\theta=1$ and $\theta=0.1$ ), the probability of reproducing (repro $=0.05$, repro $=0.01$ ), group size $(100,10)$ and survival $(\phi=0.998, \phi=0.991)$. A positive difference (on the right side of the vertical dotted line) indicates that the scenario with the higher value for a given parameter results in a higher epidemic fade-out probability than the scenario with the lower value for that parameter. This figure shows that a higher fade-out probability is most consistent for age at first between-group contact

was maintained in the population as a result of the contact between infected and susceptible juveniles from different groups.

\subsection{Emergence of the protective barrier in groups}

In the host population with communal nurseries, the proportion of groups that did not contribute to between-group transmission increased over time during the first 200 weeks after pathogen spread (Figure 5a,b), red- and blue-shaded areas. These groups were 'sealed' because they were composed of adults who were all recovered and juveniles (susceptible, infected or recovered) who did not contact members of other groups, and thus these groups did not further contribute to pathogen spread. After almost 3 years (150 weeks), all groups were sealed and composed of susceptible juveniles and recovered adults-a process which resulted in epidemic fade-out (Figures $4 \mathrm{~b}$ and $5 \mathrm{~b}$ ).

In the baseline scenario, since susceptible juveniles could become infected when contacting members of other groups, many groups remained 'open' and contributed to pathogen transmission in the host population, either by having individuals becoming infected or by having individuals infecting members of other groups. After 200 weeks, groups with susceptible juveniles and recovered adults (light blue area in Figure 5c) and groups with infected juveniles and recovered adults (light pink area in Figure $5 \mathrm{c}$ ) were both roughly equally present in the host population.

\section{DISCUSSION}

The probability of epidemic fade-out was consistently higher when juveniles had no contact with members of other groups (Figure 1-2). We specifically modelled the situation while holding $R_{0}$ similar between both scenarios and thus ignoring the direct, negative effect which reducing contacts in the communal nursery scenario would have on $R_{0}$, thus being conservative in terms of its epidemiological consequences. Our results suggest that the formation, in groups, of protective barriers composed of recovered adults around new 


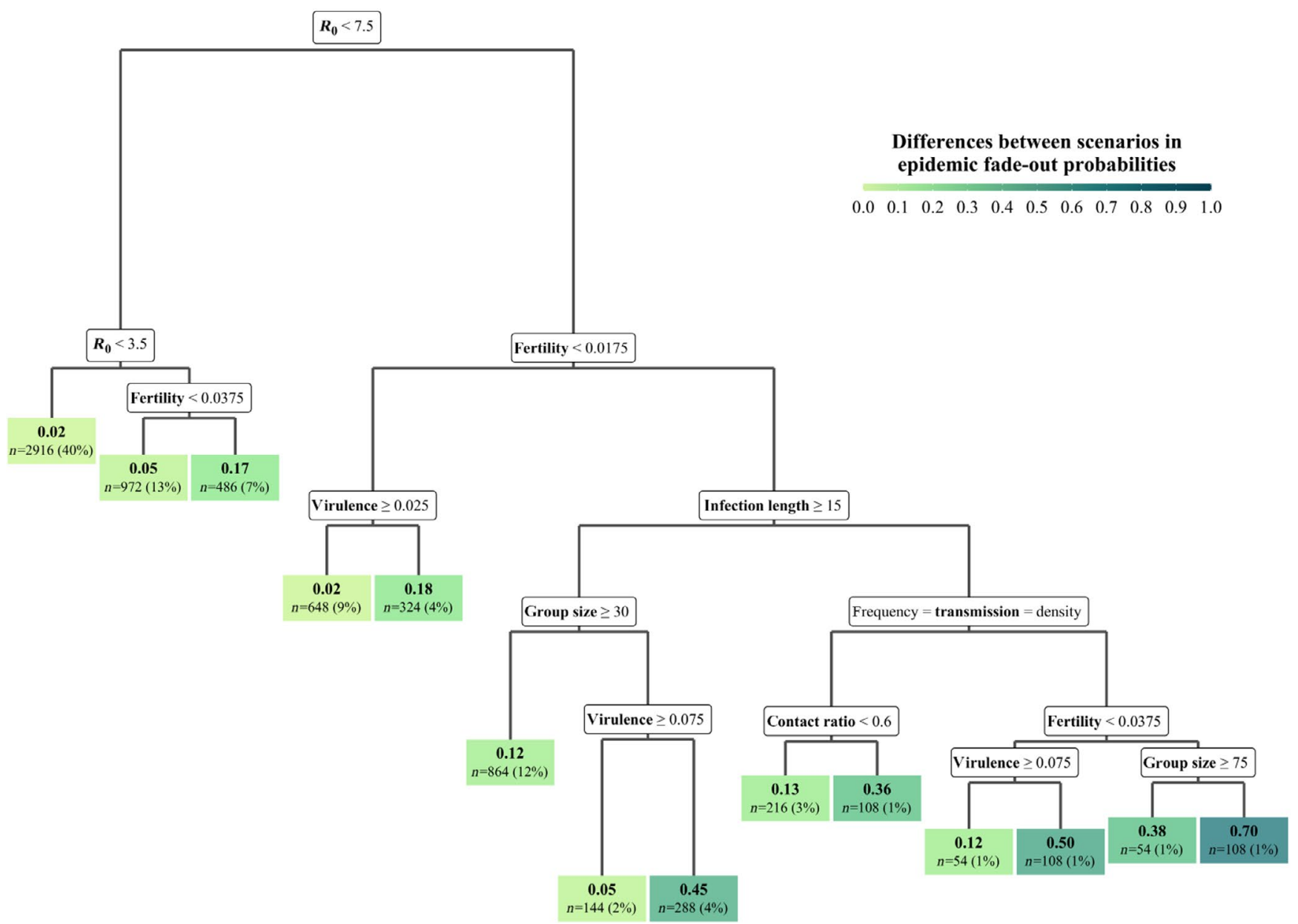

FIGURE 3 Regression tree of the differences in the epidemic fade-out probability between communal nursery and baseline scenarios, across all simulated parameters. At each node, the label indicates the parameter and the threshold to follow the left branch, for example, for $R_{0}$ the threshold was 7.5, so the left branch corresponds to $R_{0}<7.5$ and the right branch to $R_{0} \geq 7.5$. The values at the terminal nodes indicate the differences between scenarios in epidemic fade-out probabilities

generations of susceptible juveniles is a likely mechanism by which pathogen persistence is reduced in the host population (Figure $5 \mathrm{~b}$ ).

This phenomenon is expected to emerge during the late stage of an epidemic, when herd immunity-a high proportion of recovered individuals in the population (John \& Samuel, 2000)-protects the new generation of susceptible juveniles from infection (Fine et al., 2011). The complete predominance of sealed groups in host populations with communal nurseries after several weeks was associated with epidemic fade-out (Figures $4 \mathrm{~b}$ and $5 \mathrm{~b}$ ). Communal nurseries had a particularly positive effect for the host population in terms of epidemic fade-out when $R_{0}$ was high, as in measles infection in humans when $R_{0}$ may reach values of $\sim 18$ (Morris et al., 2015), and when hosts had high fertility and small group sizes (Figure 3 ). This may be because the production of many offspring in small groups may further accelerate the formation of sealed groups and hence pathogen fade-out.

We investigated a specific form of network modularity also known as 'community structure' (Edmunds et al., 1997; Longini et al., 1982; Salathé \& Jones, 2010) where the structure emerged from limited contacts between groups. Griffin and Nunn (2012) and
Nunn et al. (2015) showed that network modularity slowed pathogen spread and reduced maximum prevalence. Consistent with their results, we showed that more modular social networks reduce disease spread and persistence in group living animals when betweengroup contacts are limited in an age-dependent fashion. Sah et al. (2017) found that network modularity limited pathogen spread only when modularity was very high. Here we showed that the specific mechanism which we explored had a particularly positive effect for the host population in terms of epidemic fade-out for hosts with a high contact ratio (many between-group contacts), therefore also including networks of low modularity (Figure 3). When the contact ratio was high, the pathogen could more easily spread between groups and was less likely to die out quickly. The absence of between-group contact for juveniles was then more beneficial than when the contact ratio was low. These findings differ from those of Sah et al. (2017) possibly because we modelled host population dynamics and thus allowed modularity to develop dynamically. In our model, changes in modularity resulting from the death of infected individuals or the birth of susceptible ones constantly altered the network metrics during the epidemic-as modularity or centrality 
(a)

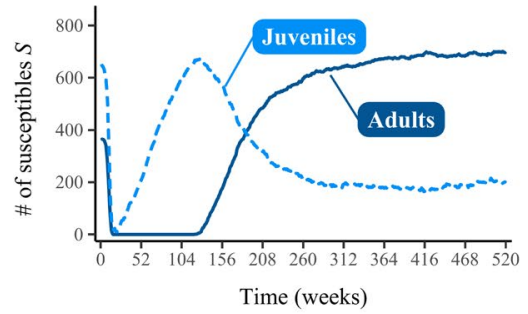

(d)

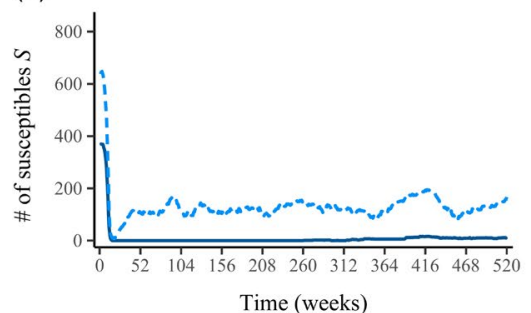

\section{Communal nursery}

(b)

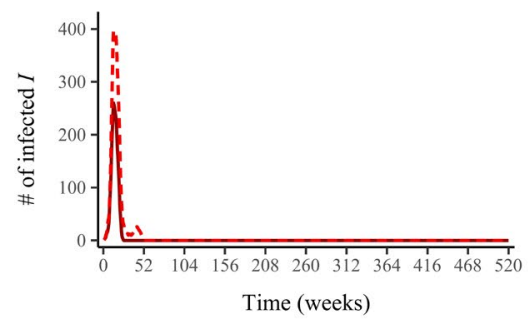

Baseline

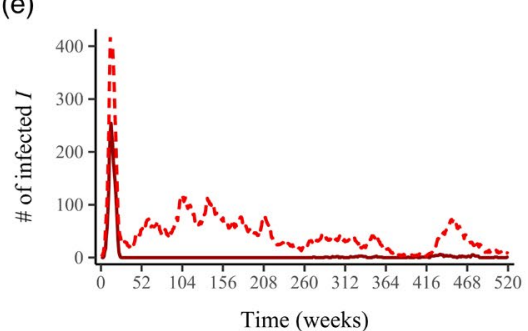

(c)

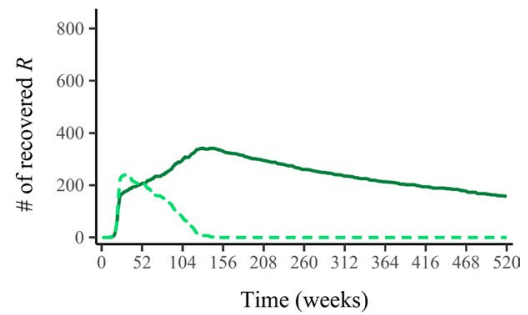

(f)

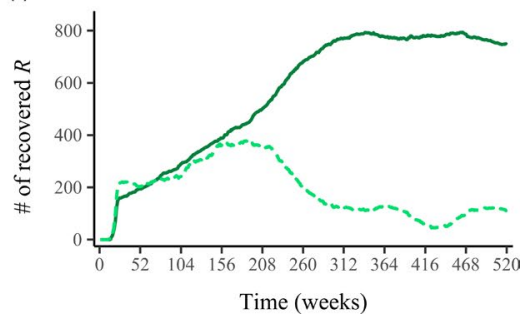

FIGURE 4 SIR dynamics during the first 10 years of pathogen spread in the communal nursery (top, a-c) and the baseline scenarios (bottom, $d-f$ ): (a) and (d) number of susceptible juveniles (dotted blue lines) and adults (full blue lines); (b) and (e) number of infected juveniles (dotted red lines) and adults (full red lines); (c) and (f): number of recovered juveniles (dotted green lines) and adults (full green lines). In both scenarios, we considered 20 groups of 50 individuals with a survival probability $\phi$ of 0.9 , a probability of reproducing repro of 0.05 , resulting in an average fecundity of 2.5 offspring per year and a within-group contact 10 times higher than the between-group contact $(\theta=0.1)$. We assumed a pathogen virulence $\alpha$ of 0.1 , an infection period infect_length of 10 weeks, an $R_{0}$ of 10 and frequency-dependent transmission. The epidemic fades out very quickly in the communal nursery scenario, whereas the population in the baseline scenario faces frequent reinfections

can only represent a snapshot of the community structure at one point in time.

In a disease management context, individuals with high contact rates to members of their own group are often targeted for vaccination to control pathogen spread (Carne et al., 2013). Our results suggest that interventions focusing on individuals with high between-group contacts could be more efficacious (Salathé \& Jones, 2010). Such individuals could be dispersing ones, as in the case of rabies in red foxes Vulpes vulpes or racoons Procyon lotor (Reynolds et al., 2015), bovine tuberculosis (bTB) in Eurasian badgers Meles meles (Weber et al., 2013) or classical swine fever (CSF) in wild boars Sus scrofa (Scherer et al., 2020). Neglecting the potentially important influence of between-group interactions on pathogen spread and enhancing the disturbance of populations through persecution and hunting may have been responsible for the failure of disease elimination programs for bTB (Vial \& Donnelly, 2011) or CSF in Germany (Scherer et al., 2019). Our results also corroborate the so-called 'network frailty concept' by demonstrating the importance of the immunisation of highly connected individuals rather than random vaccinations as a management measure (Ferrari et al., 2006).

The trait we modelled mimicked the case of hosts with clearly defined social units where juveniles are reared together, have high contact rates with members of their own group and no contact to members of other groups (see Table S1). In some mammalian carnivores, age at first between-group contact may be roughly similar to the age at which juveniles start learning hunting techniques or when juveniles go with adults on foraging trips which potentially extend beyond the territory or home range. Many social mammals where juveniles are raised in communal dens are seasonal breeders (e.g. meerkat Suricata suricatta, African wild dog Lycaon pictus, Hayssen et al., 1993). Birth patterns and the influx of new susceptible juveniles are key drivers of exposure to and infection by pathogens inducing lifelong immunity (Glass et al., 2011; Kramer-Schadt et al., 2009). For seasonal breeders, susceptible juveniles are only 'available' for infection for a few weeks or months, which might accelerate epidemic fade-out in comparison to species with year-round reproduction. Epidemic fade-out probabilities were indeed on average greater in hosts with seasonal breeding than for those with reproduction throughout the year (such as spotted hyenas, African lions Panthera leo or common vampire bats Desmodus rotundus), regardless of the scenario considered (Figure S1). The beneficial effect of communal nurseries on epidemic extinction probability is thus likely to be particularly important for species with year-round reproduction.

Social species where juveniles are not stationary but protected to some extent from between-group contacts by other types of 'barriers', such as behavioural ones, are more difficult to classify. In species with 'mobile' crèches and nurseries, such as common eider ducks Somateria mollissima, juveniles from different broods are raised together and guarded by adult females (McKinnon et al., 2006; Öst et al., 2007). In many primates such as savanna baboons Papio cynocephalus, young cling to the fur on their mother and are carried around by them for several months (Alberts, 2019). Even if juveniles 
(a)

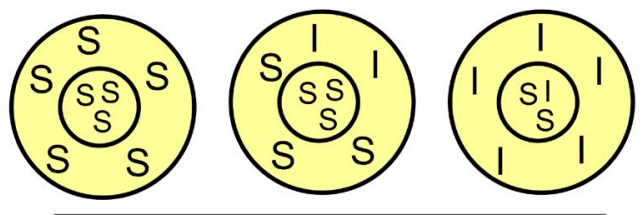

Open group

with $S$ and/or I adults
Communal nursery

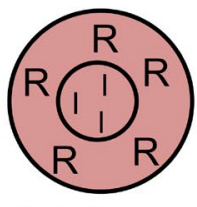

Sealed group with I juveniles

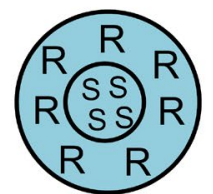

Sealed group

$R$ adults protect Sjuveniles (b)

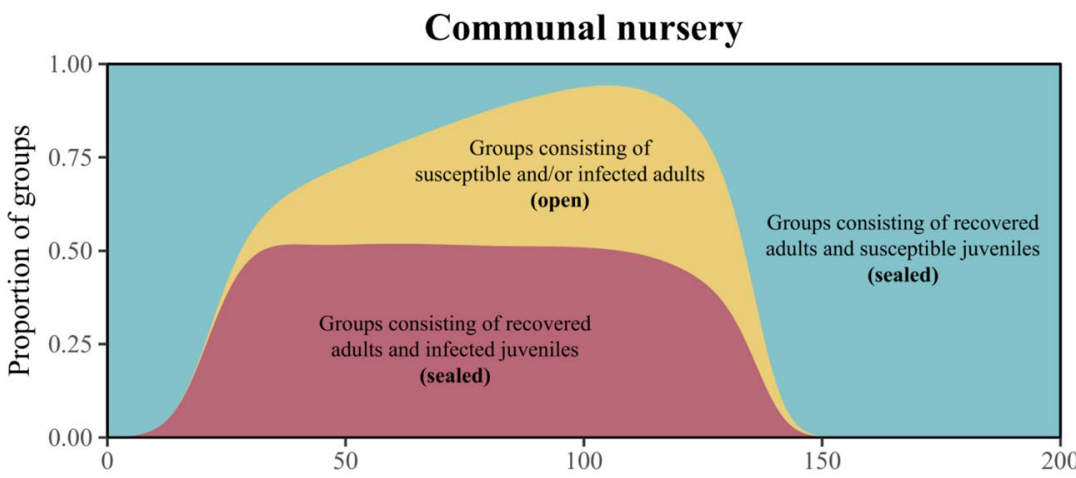

(c)

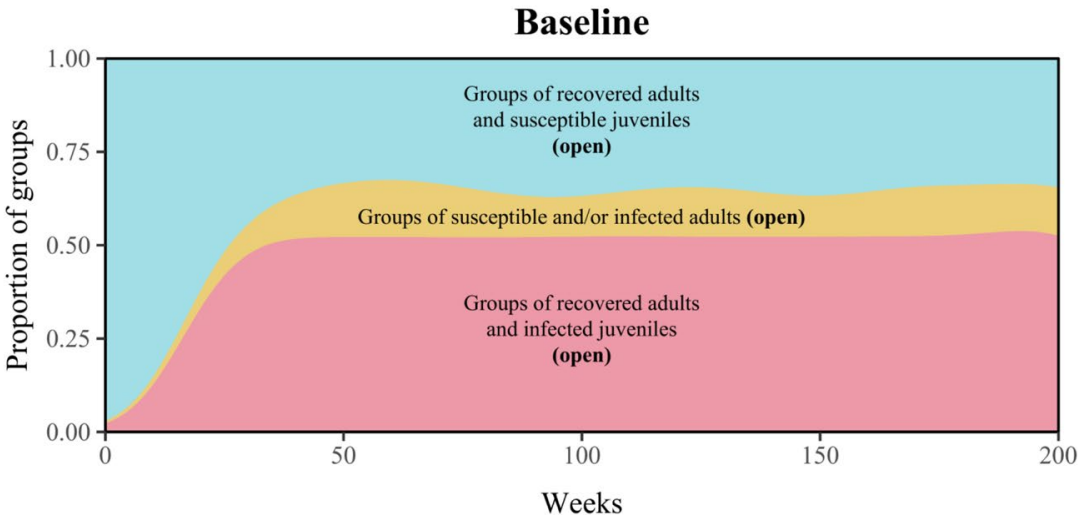

FIGURE 5 Emergence of protective barriers. Top: (a) Schematic representation of temporal changes in the composition of groups in terms of susceptible (S), infected $(\mathrm{I})$ and recovered (R) juveniles and adults, in a host population with communal nursery. Large circles represent groups and encompass both juveniles (within the small circle, small capital letters) and adults (within the outside ring, large capital letters). Bottom: Changes in the proportion of the different types of groups in the population during the course of the epidemic: (i) in yellow, groups in which all individuals are either susceptible or infected and can thus contribute to disease transmission ('open groups' in both the (b) communal nursery and (c) baseline scenarios), (ii) in pink, groups in which all adults are recovered and some juveniles are infected ('sealed groups' in (b) the communal nursery scenario and 'open groups' in (c) the baseline scenario) and (iii) in blue, groups in which all adults are recovered adults and all juveniles are susceptible ('sealed groups' in (b) the communal nursery scenario and 'open groups' in (c) the baseline scenario). We used the same trait values as for Figure 4 in such systems are unlikely to directly contact or be physically close to members of other groups during development, they may be more exposed to pathogens deposited by conspecifics in the environment (as some pathogens that transmit through contact and/or proximity may survive several hours outside their hosts) than juveniles who are stationary in communal nurseries for a relatively long proportion of their life span. Such cases could be investigated by modelling a scenario in which juveniles have lower (but non-zero) betweengroup contact levels than adults. In the absence of results from such simulations, it is unclear whether such species would benefit from increased epidemic fade-out. Given that age at first between-group contact was the parameter with the highest influence on epidemic fade-out probability (and that contact ratio also had a relatively important contribution; Figure 2), we suppose that any reduced but non-zero between-group contact would reduce heterogeneity (modularity) of contacts and thus reduce the chance of epidemic fade-out.

In our model, individuals surviving pathogen infection acquired lifelong immunity. Typical examples of such pathogens are the morbilliviruses, including the now eradicated rinderpest virus (Daszak et al., 2000) and canine distemper virus (CDV, Appel \& Summers, 1995). These morbilliviruses are highly contagious, with some strains responsible for epidemic outbreaks and high mortality in wildlife (Benhaiem et al., 2018; Marescot et al., 2018; RoelkeParker et al., 1996). CDV and related distemper viruses infect a broad range of mammals, mostly carnivores (Deem et al., 2000). Even though morbilliviruses and many other viruses are expected to induce lifelong immunity in their hosts, immunity against some viruses may wane over time or provide only incomplete protection against reinfection (Morris et al., 2015). This is often difficult to verify in free-ranging populations, as it requires either repeated serological samples to measure humoral and cellular immune responses even in old animals, or the demonstration of repeated infections through measures of repeated presence and absence of pathogen shedding (e.g. Olarte-Castillo et al., 2016). The impact of the duration of the protective period of immunisation could also be specifically tested with our model. 
The probability of epidemic fade-out was influenced by the type of pathogen transmission (Figure 3). We assumed here that the transmission mode of the pathogen is through direct contact or spatial proximity, for example, via aerosols. Although most contact-transmitted pathogens tend to be modelled as density dependent, it is now acknowledged that host-pathogen systems with frequency-dependent transmission are more prevalent in networks with a heterogeneous contact structure (Begon et al., 2002). Even in density-dependent systems, animals might seek each other out, which would result in some form of frequency-dependent transmission-be it because of aggression between dominant individuals, mate choice or other reasons. On the other hand, purely sexually transmitted pathogens could switch from frequency-dependent to density-dependent transmission when hosts become promiscuous under increasing densities (Borremans et al., 2017; Ryder et al., 2005). Clear distinctions of these two extreme forms can rarely be made under field conditions in species with complex host life histories and therefore both should be considered to take place (Borremans et al., 2017). Because the baseline scenario with homogeneous mixing (equivalent within-group and between-group contact ratios) is closer to a density-dependent transmission type, whereas communal nurseries with a low contact ratio and hence networks with high modularity reflect a frequency-dependent transmission type, we considered both transmission types in order to compare the effects of both processes on both scenarios.

To conclude, the results of our conceptual model suggest that the prolonged care of juveniles in communal nurseries can reduce pathogen persistence in host populations of group living species, particularly in species that reproduce throughout the year. Future work could focus on testing this hypothesis using parameter values from real case studies on wildlife diseases, assess how a reduction in immunity from a lifelong scenario to a scenario of immunity over a substantial proportion of the reproductive life history stage of hosts may influence the outcome and define the conditions under which this behavioural trait confers an advantage in terms of epidemic extinction probability. As discussed by White et al. (2018), we are aware that many host-pathogen systems are characterised by more complex host life histories and transmission cycles than the ones modelled here, and that dynamics may change as a function of pathogen virulence, a substantial incubation period, spillovers, the transmission of maternal antibodies to offspring, chronic infections, litter size or intermediary levels of between-group contact in juveniles. Our model also did not address the potential effects of pathogen co-infection, multi-host pathogens, indirect transmission, environmental persistence or vector foraging behaviour. Our modelling framework can easily be extended to include these more realistic and complex aspects.

\section{ACKNOWLEDGEMENTS}

The authors are very thankful to Sonja Metzger for formatting the references and to Sylvain Gandon for his advice on the model conceptualisation. This work was supported by the Leibniz Institute for Zoo and Wildlife Research. L.M. was supported by the Deutsche
Forschungsgemeinschaft (DFG) and the Agence nationale de la recherche (ANR; grants EA 5/3-1, KR 4266/2-1, ANR-16-CE02-0007), M.F. by the Leibniz-Gemeinschaft (grant SAW-2015-IZW-1 440 AquaVir), S.B. by the DFG and the Leibniz-Gemeinschaft (grants EA 5/3-1, KR 4266/2-1 and SAW-2018-IZW-3-EpiRank).

\section{CONFLICT OF INTEREST}

The authors declare no conflicts of interest.

\section{AUTHORS' CONTRIBUTIONS}

L.M., M.F., S.K.-S. and M.L.E. designed the study; L.M. and M.F. developed the IBM, and carried out the statistical analyses and drafted the manuscript with S.K.-S.; S.B. contributed critically to data interpretation, ran simulations, revised the manuscript extensively and prepared the manuscript for submission; M.L.E. and H.H. critically revised the manuscript; C.S. ran simulations, produced the figures and cleaned the final model scripts. All the authors gave final approval for publication and agreed to be held accountable for the work performed therein.

\section{DATA AVAILABILITY STATEMENT}

This article has no additional data. The model script is available on GitHub (https://github.com/EcoDynIZW/Marescot_Franz_Benha iem_2021_JAnimEcol).

\section{ORCID}

Lucile Marescot (iD https://orcid.org/0000-0001-7625-5446

Mathias Franz (iD https://orcid.org/0000-0001-5111-6503

Sarah Benhaiem (iD https://orcid.org/0000-0002-9121-5298

Cédric Scherer iD https://orcid.org/0000-0003-0465-2543

Stephanie Kramer-Schadt (iD https://orcid.

org/0000-0002-9269-4446

\section{REFERENCES}

Alberts, S. C. (2019). Social influences on survival and reproduction: Insights from a long-term study of wild baboons. Journal of Animal Ecology, 88, 47-66. https://doi.org/10.1111/1365-2656.12887

Altizer, S., Nunn, C. L., Thrall, P. H., Gittleman, J. L., Antonovics, J., Cunningham, A. A., Dobson, A. P., Ezenwa, V., Jones, K. E., Pedersen, A. B., Poss, M., \& Pulliam, J. R. C. (2003). Social organization and parasite risk in mammals: Integrating theory and empirical studies. Annual Review of Ecology, Evolution, and Systematics, 34, 517-547. https://doi.org/10.1146/annurev.ecolsys.34.030102.151725

Anderson, R. M., \& May, R. M. (1985). Vaccination and herd immunity to infectious diseases. Nature, 318, 323-329. https://doi. org/10.1038/318323a0

Anderson, R. M., \& May, R. M. (1991). Infectious diseases of humans. Dynamics and control.. Oxford University Press.

Appel, M. J., \& Summers, B. A. (1995). Pathogenicity of morbilliviruses for terrestrial carnivores. Veterinary Microbiology, 44, 187-191. https:// doi.org/10.1016/0378-1135(95)00011-X

Begon, M., Bennett, M., Bowers, R. G., French, N. P., Hazel, S. M., \& Turner, J. (2002). A clarification of transmission terms in host-microparasite models: Numbers, densities and areas. Epidemiology and Infection, 129, 147-153. https://doi.org/10.1017/S0950268802007148

Benhaiem, S., Marescot, L., East, M. L., Kramer-Schadt, S., Gimenez, O., Lebreton, J. D., \& Hofer, H. (2018). Slow recovery from a 
disease epidemic in the spotted hyena, a keystone social carnivore. Communications Biology, 1, 201. https://doi.org/10.1038/s42003018-0197-1

Borremans, B., Reijniers, J., Hens, N., \& Leirs, H. (2017). The shape of the contact-density function matters when modelling parasite transmission in fluctuating populations. Royal Society Open Science, 4. https:// doi.org/10.1098/rsos.171308

Breiman, L., Friedman, J., Olshen, R., \& Stone, C. (1984). Classification and regression trees. Wadsworth International Group, 37(15), 237-251.

Carne, C., Semple, S., Morrogh-Bernard, H., Zuberbühler, K., \& Lehmann, J. (2013). Predicting the vulnerability of great apes to disease: The role of superspreaders and their potential vaccination. PLOS ONE, 8, e84642. https://doi.org/10.1371/journal.pone.0084642

Daszak, P., Cunningham, A. A., \& Hyatt, A. D. (2000). Emerging infectious diseases of wildlife- threats to biodiversity and human health. Science, 287, 443-449. https://doi.org/10.1126/science.287.5452.443

Deem, S. L., Spelman, L. H., Yates, R. A., \& Montali, R. J. (2000). Canine distemper in terrestrial carnivores: A review. Journal of Zoo and Wildlife Medicine, 31, 441-451. https://doi.org/10.1638/10427260(2000)031[0441:cditca]2.0.co;2

Edmunds, W. J., O'callaghan, C. J., \& Nokes, D. J. (1997). Who mixes with whom? A method to determine the contact patterns of adults that may lead to the spread of airborne infections. Proceedings of the Royal Society of London, Series B: Biological Sciences, 264, 949-957. https:// doi.org/10.1098/rspb.1997.0131

Ferrari, M. J., Bansal, S., Meyers, L. A., \& Bjørnstad, O. N. (2006). Network frailty and the geometry of herd immunity. Proceedings of the Royal Society B: Biological Sciences, 273, 2743-2748. https://doi. org/10.1098/rspb.2006.3636

Fine, P., Eames, K., \& Heymann, D. L. (2011). 'Herd immunity': A rough guide. Clinical Infectious Diseases, 52, 911-916. https://doi. org/10.1093/cid/cir007

Glass, K., Mercer, G. N., Nishiura, H., McBryde, E. S., \& Becker, N. G. (2011). Estimating reproduction numbers for adults and children from case data. Journal of the Royal Society, Interface, 8, 1248-1259. https://doi.org/10.1098/rsif.2010.0679

Griffin, R. H., \& Nunn, C. L. (2012). Community structure and the spread of infectious disease in primate social networks. Evolutionary Ecology, 26, 779-800. https://doi.org/10.1007/s10682-011-9526-2

Grimm, V., Berger, U., Bastiansen, F., Eliassen, S., Ginot, V., Giske, J., Goss-Custard, J., Grand, T., Heinz, S. K., Huse, G., Huth, A., Jepsen, J. U., Jørgensen, C., Mooij, W. M., Müller, B., Pe'er, G., Piou, C., Railsback, S. F., Robbins, A. M., ... DeAngelis, D. L. (2006). A standard protocol for describing individual-based and agent-based models. Ecological Modelling, 198, 115-126. https://doi.org/10.1016/j.ecolm odel.2006.04.023

Grimm, V., Berger, U., DeAngelis, D. L., Polhill, J. G., Giske, J., \& Railsback, S. F. (2010). The ODD protocol: A review and first update. Ecological Modelling, 221, 2760-2768. https://doi.org/10.1016/j.ecolmodel. 2010.08.019

Hayssen, V., van Tienhoven, A., \& van Tienhoven, A. (1993). Asdell's patterns of mammalian reproduction: A compendium of species-specific data. Cornell University Press.

John, T. J., \& Samuel, R. (2000). Herd immunity and herd effect: New insights and definitions. European Journal of Epidemiology, 16, 601-606. https://doi.org/10.1023/A:1007626510002

Kappeler, P. M., Cremer, S., \& Nunn, C. L. (2015). Sociality and health: Impacts of sociality on disease susceptibility and transmission in animal and human societies. Philosophical Transactions of the Royal Society B: Biological Sciences, 370, 20140116. https://doi. org/10.1098/rstb.2014.0116

Kramer-Schadt, S., Fernández, N., Eisinger, D., Grimm, V., \& Thulke, H. H. (2009). Individual variations in infectiousness explain long-term disease persistence in wildlife populations. Oikos, 118, 199-208. https://doi.org/10.1111/j.1600-0706.2008.16582.x
Kruuk, H. (1972). The spotted hyena: A study of predation and social behavior. University of Chicago Press.

Lloyd-Smith, J. O., Schreiber, S. J., Kopp, P. E., \& Getz, W. M. (2005) Superspreading and the effect of individual variation on disease emergence. Nature, 438, 355-359. https://doi.org/10.1038/natur e04153

Longini Jr., I. M., Koopman, J. S., Monto, A. S., \& Fox, J. P. (1982). Estimating household and community transmission parameters for influenza. American Journal of Epidemiology, 115, 736-751. https:// doi.org/10.1093/oxfordjournals.aje.a113356

Marescot, L., Benhaiem, S., Gimenez, O., Hofer, H., Lebreton, J. D., Olarte-Castillo, X. A., Kramer-Schadt, S., \& East, M. L. (2018). Social status mediates the fitness costs of infection with canine distemper virus in Serengeti spotted hyenas. Functional Ecology, 32, 1237-1250. https://doi.org/10.1111/1365-2435.13059

McKinnon, L., Gilchrist, H. G., \& Scribner, K. T. (2006). Genetic evidence for kin-based female social structure in common eiders (Somateria mollissima). Behavioral Ecology, 17, 614-621. https://doi.org/10.1093/ beheco/ark002

Mech, L. D., \& Boitani, L. (2003). Wolves: Behavior, ecology, and conserva tion. University of Chicago Press.

Morris, S. E., Pitzer, V. E., Viboud, C., Metcalf, C. J. E., Bjørnstad, O. N., $\&$ Grenfell, B. T. (2015). Demographic buffering: Titrating the effects of birth rate and imperfect immunity on epidemic dynamics. Journal of the Royal Society, Interface, 12, 20141245. https://doi.org/10.1098/ rsif.2014.1245

Müller, K. (2017). here: A simpler way to find your files. R package version 0.1. Retrieved from HYPERLINK "sps:urlprefix::https" HYPERLINK "sps:urlprefix::https" HYPERLINK "sps:urlprefix::https" https:// CRAN.R-project.org/package=here

Newman, M. E. J. (2006). Modularity and community structure in networks. Proceedings of the National Academy of Sciences of the United States of America, 103, 8577-8582. https://doi.org/10.1073/ pnas.0601602103

Nunn, C. L., Jordán, F., McCabe, C. M., Verdolin, J. L., \& Fewell, J. H. (2015). Infectious disease and group size: More than just a numbers game. Philosophical Transactions of the Royal Society B: Biological Sciences, 370, 20140111. https://doi.org/10.1098/rstb.2014.0111

Nunn, M. A., Barton, T. R., Wanless, S., Hails, R. S., Harris, M. P., \& Nuttall, P. A. (2006). Tick-borne Great Island Virus: (II) Impact of age-related acquired immunity on transmission in a natural seabird host. Parasitology, 132, 241. https://doi.org/10.1017/S003118200 5008954

Olarte-Castillo, X. A., Hofer, H., Goller, K. V., Martella, V., Moehlman, P. D., \& East, M. L. (2016). Divergent Sapovirus-like strains and infection prevalence in wild carnivores in the Serengeti ecosystem. PLoS ONE, 11, e0163548. https://doi.org/10.1371/journal.pone.0163548

Öst, M., Clark, C. W., Kilpi, M., \& Ydenberg, R. (2007). Parental effort and reproductive skew in coalitions of brood-rearing female common eiders. The American Naturalist, 169, 73-86. https://doi. org/10.1086/510213

Pedersen, T. L. (2019). patchwork: The composer of plots. R package version 1.0.0. Retrieved from https://CRAN.R-project.org/package=patchwork

Qiu, Y. (2020). showtext: Using fonts more easily in $R$ graphs. $R$ package version 0.7-1. Retrieved from https://CRAN.R-project.org/packa ge=showtext

R Core Team. (2020). R: A language and environment for statistical computing. R Foundation for Statistical Computing.

Reynolds, J. J., Hirsch, B. T., Gehrt, S. D., \& Craft, M. E. (2015). Raccoon contact networks predict seasonal susceptibility to rabies outbreaks and limitations of vaccination. Journal of Animal Ecology, 84, 17201731. https://doi.org/10.1111/1365-2656.12422

Roelke-Parker, M. E., Munson, L., Packer, C., Kock, R., Cleaveland, S., Carpenter, M., O'Brien, S. J., Pospischil, A., Hofmann-Lehmann, R., 
Lutz, H., Mwamengele, G. L. M., Mgasa, M. N., Machange, G. A., Summers, B. A., \& Appel, M. J. G. (1996). A canine distemper virus epidemic in Serengeti lions (Panthera leo). Nature, 379, 441-445. https://doi.org/10.1038/379441a0

RStudio Team. (2020). RStudio: Integrated Development Environment for $R$. RStudio, PBC. Retrieved from http://www.rstudio.com/

Ryder, J. J., Webberley, K. M., Boots, M., \& Knell, R. J. (2005). Measuring the transmission dynamics of a sexually transmitted disease. Proceedings of the National Academy of Sciences of the United States of America, 102, 15140-15143. https://doi.org/10.1073/pnas.05051 39102

Sah, P., Leu, S. T., Cross, P. C., Hudson, P. J., \& Bansal, S. (2017). Unraveling the disease consequences and mechanisms of modular structure in animal social networks. Proceedings of the National Academy of Sciences of the United States of America, 114, 4165-4170. https://doi. org/10.1073/pnas.1613616114

Salathé, M., \& Jones, J. H. (2010). Dynamics and control of diseases in networks with community structure. PLoS Computational Biology, 6, e1000736. https://doi.org/10.1371/journal.pcbi.1000736

Scherer, C., Radchuk, V., Franz, M., Thulke, H., Lange, M., Grimm, V., \& Kramer-Schadt, S. (2020). Moving infections: Individual movement decisions drive disease persistence in spatially structured landscapes. Oikos, https://doi.org/10.1111/oik.07002

Scherer, C., Radchuk, V., Staubach, C., Müller, S., Blaum, N., Thulke, H. H., \& Kramer-Schadt, S. (2019). Seasonal host life-history processes fuel disease dynamics at different spatial scales. Journal of Animal Ecology, 88, 1812-1824. https://doi.org/10.1111/1365-2656.13070

Stroeymeyt, N., Grasse, A. V., Crespi, A., Mersch, D. P., Cremer, S., \& Keller, L. (2018). Social network plasticity decreases disease transmission in a eusocial insect. Science, 362, 941-945. https://doi. org/10.1126/science.aat4793

Therneau, T., \& Atkinson, B. (2018). rpart: Recursive partitioning and regression trees. R package version 4.1-13. Retrieved from https:// CRAN.R-project.org/package $=$ rpart

Vial, F., \& Donnelly, C. A. (2011). Localized reactive badger culling increases risk of bovine tuberculosis in nearby cattle herds. Biology Letters, 8, 50-53. https://doi.org/10.1098/rsbl.2011.0554

Wanelik, K. M., Burthe, S. J., Harris, M. P., Nunn, M. A., Godfray, H. C. J., Sheldon, B. C., McLean, A. R., \& Wanless, S. (2017). Investigating the effects of age-related spatial structuring on the transmission of a tick-borne virus in a colonially breeding host. Ecology and Evolution, 7, 10930-10940. https://doi.org/10.1002/ece3.3612
Weber, N., Carter, S. P., Dall, S. R., Delahay, R. J., McDonald, J. L., Bearhop, S., \& McDonald, R. A. (2013). Badger social networks correlate with tuberculosis infection. Current Biology, 23, 915-916. https://doi. org/10.1016/j.cub.2013.09.011

Wey, T. W., \& Blumstein, D. T. (2010). Social cohesion in yellowbellied marmots is established through age and kin structuring. Animal Behavior, 79, 1343-1352. https://doi.org/10.1016/j.anbeh av.2010.03.008

White, L. A., Forester, J. D., \& Craft, M. E. (2018). Disease outbreak thresholds emerge from interactions between movement behavior, landscape structure, and epidemiology. Proceedings of the National Academy of Sciences of the United States of America, 115, 7374-7379. https://doi.org/10.1073/pnas.1801383115

Wickham, H. (2016). ggplot2: Elegant graphics for data analysis. Springer International Publishing. https://doi.org/10.1007/978-3319-24277-4

Wickham, H., Averick, M., Bryan, J., Chang, W., McGowan, L., François, R., Grolemund, G., Hayes, A., Henry, L., Hester, J., Kuhn, M., Pedersen, T., Miller, E., Bache, S., Müller, K., Ooms, J., Robinson, D., Seidel, D., Spinu, V., ... Yutani, H. (2019). Welcome to the Tidyverse. Journal of Open Source Software, 4(43), 1686. https://doi.org/10.21105/ joss.01686

Wickham, H., François, R., Henry, L., \& Müller, K. (2019). dplyr: A grammar of data manipulation. $\mathrm{R}$ package version 0.8.3. Retrieved from https:// CRAN.R-project.org/package $=$ dplyr

Wilke, C. O. (2020). ggtext: Improved text rendering support for 'ggplot2'. $\mathrm{R}$ package version 0.1.0. Retrieved from https://wilkelab.org/ggtext

\section{SUPPORTING INFORMATION}

Additional supporting information may be found online in the Supporting Information section.

How to cite this article: Marescot, L., Franz, M., Benhaiem, S., Hofer, H., Scherer, C., East, M. L., \& Kramer-Schadt, S. (2021). 'Keeping the kids at home' can limit the persistence of contagious pathogens in social animals. Journal of Animal Ecology, 90, 2523-2535. https://doi.org/10.1111/1365-2656.13555 